\title{
Multicolour photometry of the M0V+M5V eclipsing binary V405 And
}

\author{
K. Vida*,†, K. Oláh ${ }^{\dagger}$, Zs. Kővári ${ }^{\dagger}$ and J. Bartus** \\ *Eötvös Loránd University, Department of Astronomy, Budapest,Hungary \\ ${ }^{\dagger}$ Konkoly Observatory, Budapest, Hungary \\ ${ }^{* *}$ Astrophysikalisches Institut Potsdam, Germany
}

\begin{abstract}
We present multicolour photometry and modelling of the active eclipsing binary star V405 And. The components of 0.2 and 0.5 solar masses are just below and above the theoretical limit of the full convection, that is thought to be around 0.3 solar mass. The light curves are compositions of constant and variable features: the distorted shape of the components (about 25\%), a small eclipse, and mainly of spots (about $75 \%$ ) and flares.
\end{abstract}

Keywords: Stars: activity,Stars: starspots, Stars: flare, (Stars:) binaries: eclipsing

PACS: 97.10.Jb, 97.10.Qh, 97.20.Jg, 97.80.Hn

\section{INTRODUCTION}

The manifestations of stellar activity appearing as spots, plages, flares, activity cycles etc., are consequences of the strong magnetic fields. The origin of this magnetic field of stars is some kind of a dynamo mechanism, which can be the so-called $\alpha \Omega$ dynamo, based on the amplification of the fields by differential rotation in the tachocline, which is a thin interface layer between the convection zone and the radiative core. Less massive stars are thought to be fully convective, wherein the $\alpha^{2}$ dynamo generates strong, longlasting, axisymmetric magnetic fields. The mass limit for full convection is thought to be around 0.35 solar mass. The late type binary V405 And is an excellent target to study the effects of the two possible dynamos, since one component is below, the other is above the mass limit of the full convection [1].

\section{OBSERVATIONS AND DATA REDUCTION}

Observations were carried out using the 1m RCC-telescope of the Konkoly Observatory at Piszkéstetô Mountain Station. The telescope is equipped with a Princeton Instruments $1300 \times 1300 \mathrm{CCD}$. We present data obtained on 5 nights (between JD 24543732454426). We also present one night of observation (at JD 2454141) obtained with the 60 cm telescope of the Konkoly Observatory, Svábhegy, Budapest equipped with a Wright Instruments $750 \times 1100 \mathrm{CCD}$ camera. For differential photometry GSC 03298-00148 was used as comparison, GSC 03298-01110 as check star. Data reduction was done us- 


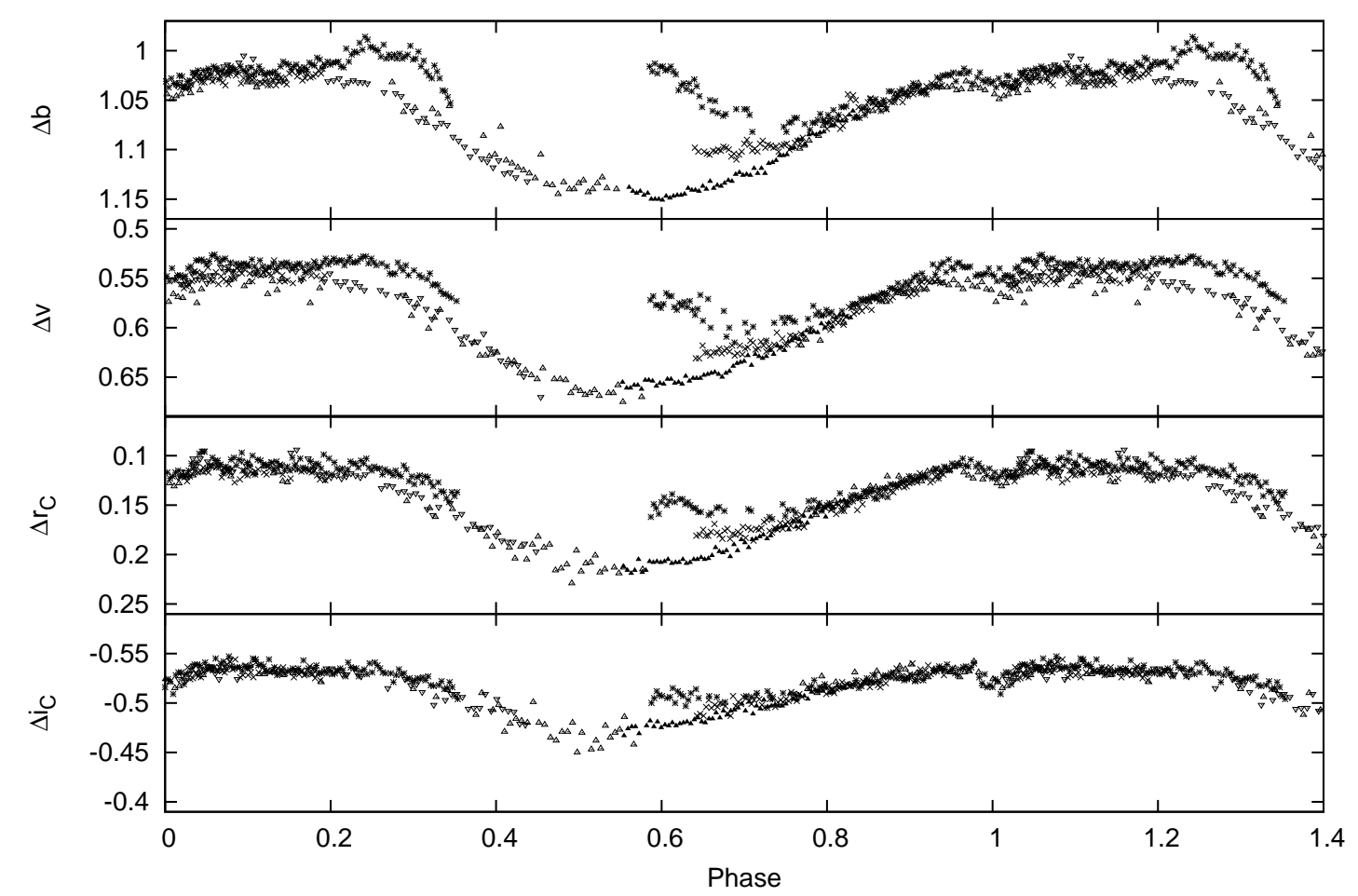

FIGURE 1. Observational data obtained with the $1 \mathrm{~m} \mathrm{RCC}$ telescope in Konkoly Observatory. Each observed night was plotted with different symbols. Note the small eclipse at phase 1.0. The light curve show the slow flare observed on JD 2454373 and JD 2454374 starting at phase $\approx 0.6$. (see Fig. 2b)

ing standard IRAF ${ }^{1}$ packages and DAOPHOT was used for photometry. The resulting light curve is plotted in Fig. 1.

\section{ANALYSIS OF THE DATA}

For modelling the data, we used the light curve between HJD 245422-245426 from the RCC telescope, free from flare activity. From Fig. 5 in [1] it is seen that the light curve of V405 And is quite stable, and since our data used for eclipse modelling is observed within 5 days, the effect of spot changes, thus the modulation of the light curve is probably small. Since the light curve is composed of eclipse/shape distortion and of modulation caused by starspots, parallel modelling of both effects is needed [2]. Basic binary parameters (semi-major axis, mass ratio, orbital period) were known from the radial velocity curve [1], thus we had good assumptions on stellar radii and system inclination. Temperatures were estimated from the spectral types using the tables of [3]

${ }^{1}$ IRAF is distributed by the National Optical Astronomy Observatory, which is operated by the Association of Universities for Research in Astronomy, Inc., under cooperative agreement with the National Science Foundation. 
TABLE 1. System parameters for V405 And

\begin{aligned} \hline$P_{\text {orb }}=P_{\text {rot }} & =0.465$ days* \\ $\mathrm{a} & =2.246 R_{\odot}{ }^{*} \\ i & =66.5 \\ M_{1} & =0.495 M_{\odot} \\ M_{2} & =0.208 M_{\odot} \\ T_{1} & =4050 \mathrm{~K} \\ T_{2} & =3000 \mathrm{~K} \\ R_{1} & =0.785 R_{\odot} \\ R_{2} & =0.235 R_{\odot} \\ v \sin i & =85 \mathrm{~km} / \mathrm{s} \\ e & =0.0^{*}\end{aligned}$

* taken from [1]

and [4]. The binary solution was carried out using PHOEBE [5]. The derived system parameters are summarised in Table 1. A spot model has been calculated using the program SML (SpotModeL, [6]) assuming the presence of two cool circular spots with $T_{\text {spot }}=3300 \mathrm{~K}$, on the primary star. This assumption is plausible, since the spottedness of the smaller, fainter companion wouldn't affect the shape of the light curve much.

The light curve solution of the was carried out in the following way: first, a binary model is substracted from the observed light curve. Since the resulting light curve is free from the effects of the binarity - the eclipse and the distorted shape, the only cause of the changes is the spottedness of the primary star. In the next step a spot model is applied, which is then removed from the original, observed light curve. The result is an unspotted binary light curve, for which a more precise binary model can be derived. After some iterations times the light curve can be fitted with a composite solution of the spots and binarity. During the procedure the unspotted brightness of the system should be taken into account, which, in our case is the brightest magnitude ever observed [1].

In the observations, two remarkable flare events occurred. The light curves of the flares are plotted in Fig. 2, where the binary and spot models are substracted. The estimated flare energies for the larger one appeared at phase $\approx 0.8$ (Fig. 2a) in $B, V, R_{C}$ and $I_{C}$ passbands are $14.10 \times 10^{35}, 7.38 \times 10^{35}, 13.33 \times 10^{35}$ and $13.25 \times 10^{35} \mathrm{ergs} / \mathrm{sec}$, respectively (for the description of the estimation method, see e.g. [7]).

In the case of the second, much slower flare event, starting at phase $\approx 0.6$ (Fig. 2b) the phenomenon remains observable for at least 3 rotations. During this time, the flaring region moves out of view at phase $\approx 0.9$.

\section{ACKNOWLEDGMENTS}

$\mathrm{KV}, \mathrm{KO}$ and are supported by the Hungarian Science Research Program (OTKA) grant T-048961. ZsK is a grantee of the Bolyai János Scholarship of the Hungarian Academy of Sciences. 

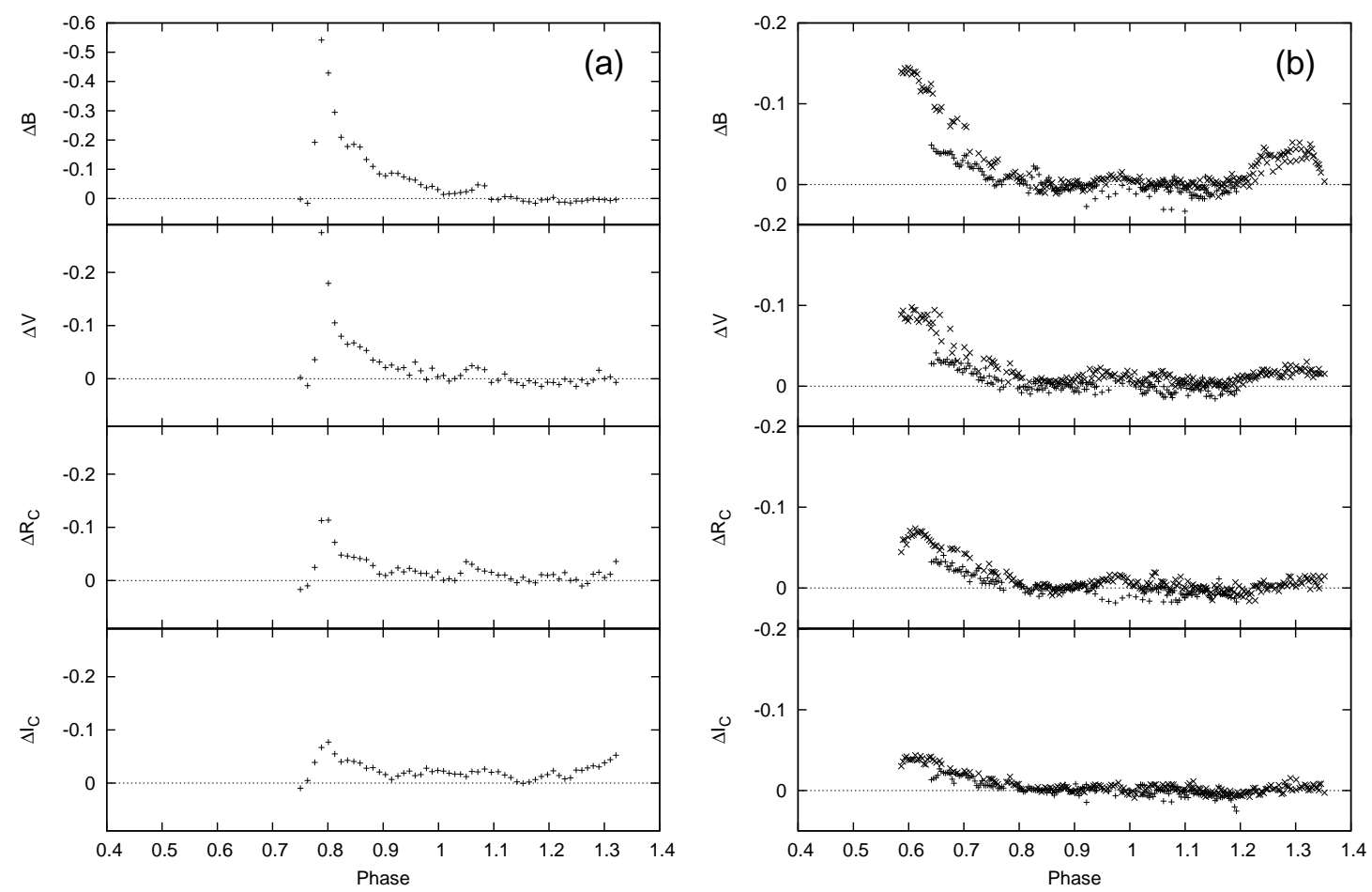

FIGURE 2. (a) A giant flare observed at HJD 2454141 using the $60 \mathrm{~cm}$ telescope of Konkoly Observatory. (b) A long lasting (at least 3 days) slow flare event from the $1 \mathrm{~m} \mathrm{RCC}$ data observed at HJD 2454373 (plus signs) and at HJD 2454374 (crosses). Binary+spot model light curves are subtracted from the original data, a line represents the undisturbed stellar surface.

\section{REFERENCES}

1. C. Chevalier, and S. A. Ilovaisky, A\&A 326, 228-236 (1997), arXiv: astro-ph/ 9705018.

2. K. G. Strassmeier, J. Bartus, F. C. Fekel, and G. W. Henry, A\&A 485, 233-243 (2008).

3. P. J. Flower, ApJ 469, 355-365 (1996).

4. D. A. VandenBerg, and J. L. Clem, AJ 126, 778-802 (2003).

5. A. Prša, and T. Zwitter, ApJ 628, 426-438 (2005), arXiv : ast ro-ph / 0503361.

6. G. Ribárik, K. Oláh, and K. G. Strassmeier, Astronomische Nachrichten 324, 202-214 (2003).

7. Z. Kővári, F. Vilardell, I. Ribas, K. Vida, L. van Driel-Gesztelyi, C. Jordi, and Oláh, K., Astronomische Nachrichten 328, 904-908 (2007), arXiv : 0711.0742. 